\title{
Joint Localization and Time Synchronization in Wireless Sensor Networks with Anchor Uncertainties
}

\author{
Jun Zheng and Yik-Chung Wu \\ Department of Electrical and Electronic Engineering \\ The University of Hong Kong, Hong Kong \\ Email: \{zhengjun, ycwu\}@eee.hku.hk
}

\begin{abstract}
Although localization and synchronization share many aspects in common, they are traditionally treated separately. In this paper, we present a unified framework to jointly solve these two problems at the same time. The joint approach is attractive because it can solve both localization and synchronization using the same set of message exchanges. This is extremely important for energy saving, especially for the energy constrained wireless sensor networks. Furthermore, since the accuracy of localization and synchronization is very sensitive to the accuracy of anchor locations and timings, the joint localization and synchronization problem with inaccurate anchor is considered in this paper. A novel generalized total least squares (GTLS) based method is proposed and the Cramer-Rao lower bound (CRLB) for the joint localization and time synchronization is derived. Simulation results show that the mean square error performances of the proposed estimator can attain the CRLB.
\end{abstract}

\section{INTRODUCTION}

The rapid advances in micro-electro-mechanical systems make it possible to produce a large number of low-cost, low-power and multi-functional tiny sensor nodes, and thus propels the implementation of modern large scale wireless sensor networks (WSNs). Because of its wide applications in environmental monitoring, natural disaster prediction, health care, manufacturing and transportation, WSNs have attracted enormous interest in recent years [1], [2].

In WSNs, localization is the basis of applications which require the accurate locations of the sensor nodes, such as environment monitoring, target and event tracking, emergency rescue and geographic routing. On the other hand, synchronization supports functions such as time-based channel sharing, power scheduling, and time-based localization in WSNs [1].

While localization is traditionally studied from the signal processing point of view [3], synchronization is mainly studied from protocol design of view [4]. As a result, these two problems have been investigated separately for a long time. However, localization and synchronization have very close relationships and share many aspects in common. For timebased (such as TOA) localization algorithms, synchronization is even a prerequisite [3].

Based on the close relationships between localization and time synchronization, it is natural to explore the possibility of formulating them into a unified framework and solve the two problems at the same time. The joint localization and synchronization approach is extremely attractive in WSNs because the joint approach makes it possible to carry out localization and synchronization with only one set of data package exchanges, rather than two. This is extremely crucial for WSNs as the power and memory of the sensor nodes are very limited.

Recently, some pioneering research works noticed the similarities between the problem of localization and time synchronization [5]. However, [5] only explores the possibility of jointly implementing localization and synchronization at the protocol level. In [6], it was the first time a unified framework for joint localization and time synchronization was proposed from signal processing perspective.

In this paper, the results in [6] are extended to the case where there are uncertainties in anchor locations and timings. In WSNs, hierarchical method is usually used to localize and synchronize a large sensor field [1], [7]. Some sensor nodes are localized and synchronized to current anchors, and they become new anchors and are used to localize and synchronize other nodes. This procedure is repeated until all sensor nodes are localized and synchronized. A problem with this hierarchical localization and synchronization method is the error propagation due to the inaccuracy of estimations at each level. In this case, taking the uncertainties in anchor locations and timings is important for error propagation relief. In this paper, both location and timing uncertainties of anchors are incorporated in the system model, and a novel estimator is proposed based on the generalized total least squares (GTLS) method.

Notations: The following notations are used in this paper. Matrices $\mathbf{1}_{M \times N}, \mathbf{0}_{M \times N}, \boldsymbol{I}_{M}$ and $\boldsymbol{E}_{M \times 1}$ denote the $M \times N$ matrix of ones, $M \times N$ matrix of zeros, $M \times M$ identity matrix, and an $M \times 1$ vector of alternating -1 and 1 , respectively. The operators $\otimes, \odot, \operatorname{Tr}(\cdot)$ and $\{\cdot\}^{T}$ denote the Kronecker product, the Hadamard product, the trace of a square matrix and the transpose of a matrix, respectively. The Euclidean norm and Frobenius norm are denoted as $\|\cdot\|$ and $\|\cdot\|_{F}$, respectively, while $|\cdot|$ is the absolute of a scalar or the determinant of a matrix. The operator $E\{\cdot\}$ is the expectation of a random 
variable or matrix, $\operatorname{diag}\{\boldsymbol{A}, \boldsymbol{B}\}$ is a matrix with $\boldsymbol{A}$ and $\boldsymbol{B}$ on its diagonal and all other elements zero, $\mathcal{R}(\cdot)$ is the range space of a matrix, and $C^{1 / 2}$ is the Cholesky decomposition of $C$.

\section{SySTEM MODEL}

We consider a single node joint localization and synchronization in a WSN, where only one node needs to be localized and synchronized to the anchors at a time. In this system, it is assumed that there are $L(L \geq 3)$ anchors with known locations and timings. The $l^{\text {th }}$ anchor $A_{l}$ is located at $\boldsymbol{a}_{l}^{o}=$ $\left[a_{x l}^{o}, a_{y l}^{o}\right]^{T}$ with time skew $\theta_{s l}^{o}$ and time offset $\theta_{0 l}^{o}$. A Node $B$ with unknown location $\boldsymbol{x}=[x, y]^{T}$, time skew $\theta_{s}$ and time offset $\theta_{0}$ exchanges time-stamped packages with anchors and uses the exchanged time-stamps to estimate its location and timing parameters.

The information communicated between the node and the anchors is the time-stamps in two-way message exchanges [8]. It is assumed that there are $M$ rounds of time-stamp exchanges between Node $B$ and anchor $A_{l}$. As is shown in Figure 1, the $m^{t h}$ message is sent from Node $B$ at time $T_{l m}$ and is received by $A_{l}$ at time $R_{l m}$. Then, anchor $A_{l}$ replies Node $B$ with another message sent at $\bar{T}_{l m}$ and is received by Node $B$ at $\bar{R}_{l m}$. In the reply message from anchor $A_{l}$ to Node $B$, the time-stamps $R_{l m}$ and $\bar{T}_{l m}$ at the anchor side are also included. Therefore, Node $B$ has all the time-stamp information $\left\{T_{l m}, R_{l m}, \bar{T}_{l m}, \bar{R}_{l m}\right\}$. Note that $R_{l m}$ and $\bar{T}_{l m}$ are measured with respect to the clock of anchors, while $T_{l m}$ and $\bar{R}_{l m}$ are measured with respect to the clock of Node $B$. The exchanged time-stamps can be modeled as [9]

$$
\begin{aligned}
& T_{l m}=\frac{\theta_{s}}{\theta_{s l}^{o}} R_{l m}-\theta_{s}\left(t_{l}+n_{l m}\right)+\theta_{0}-\frac{\theta_{s}}{\theta_{s l}^{o}} \theta_{0 l}^{o}, \\
& \bar{R}_{l m}=\frac{\theta_{s}}{\theta_{s l}^{o}} \bar{T}_{l m}+\theta_{s}\left(t_{l}+\bar{n}_{l m}\right)+\theta_{0}-\frac{\theta_{s}}{\theta_{s l}^{o}} \theta_{0 l}^{o},
\end{aligned}
$$

where $t_{l}=\left\|\boldsymbol{x}-\boldsymbol{a}_{l}^{o}\right\| / c$ is the propagation delay between Node $B$ and anchor $A_{l}$, with $c$ being the speed of light. Symbols $n_{l m}$ and $\bar{n}_{l m}$ are the TOA detection errors, which are Gaussian distributed [10], with zero mean and variance $\sigma_{n}^{2}$. With the impulse-radio ultra-wideband technology and physical layer time-stamping, the TOA detection errors are in the order of nanosecond (ns) [11].

When there are anchor uncertainties, we can only have the observed (but not true) values of the location, time skew and time offset of anchors, i.e.,

$$
\boldsymbol{a}_{l}=\boldsymbol{a}_{l}^{o}-\Delta \boldsymbol{a}_{l}, \theta_{s l}=\theta_{s l}^{o}-\Delta \theta_{s l}, \theta_{0 l}=\theta_{0 l}^{o}-\Delta \theta_{0 l},
$$

where $\Delta \boldsymbol{a}_{l}=\left[\Delta a_{x l}, \Delta a_{y l}\right]^{T}, \Delta \theta_{s l}$ and $\Delta \theta_{0 l}$ are the uncertainties in anchor location, time skew and time offset, respectively. Since the timing and location uncertainties of anchors usually arise from different sources, it is assumed that $\Delta \boldsymbol{a}_{l}, \Delta \theta_{s l}$ and $\Delta \theta_{0 l}$ are independent and identically distributed (i.i.d.) Gaussian with zero means and variances of $\sigma_{a}^{2}, \sigma_{s}^{2}$ and $\sigma_{o}^{2}$, respectively.
Taking anchor uncertainties into account, the exchanged time-stamps in (1) and (2) become

$$
\begin{aligned}
-T_{l m}=-\frac{\theta_{s}}{\theta_{s l}+\Delta \theta_{s l}} R_{l m}+\theta_{s}\left(t_{l}+n_{l m}\right) & \\
- & \theta_{0}+\frac{\theta_{s}}{\theta_{s l}+\Delta \theta_{s l}}\left(\theta_{0 l}+\Delta \theta_{0 l}\right), \\
\bar{R}_{l m}=\frac{\theta_{s}}{\theta_{s l}+\Delta \theta_{s l}} & \bar{T}_{l m}+\theta_{s}\left(t_{l}+\bar{n}_{l m}\right) \\
& \quad \theta_{0}-\frac{\theta_{s}}{\theta_{s l}+\Delta \theta_{s l}}\left(\theta_{0 l}+\Delta \theta_{0 l}\right),
\end{aligned}
$$

where $\theta_{s l}^{o}$ and $\theta_{0 l}^{o}$ in (1) and (2) were replaced by $\theta_{s l}+\Delta \theta_{s l}$ and $\theta_{0 l}+\Delta \theta_{0 l}$, respectively.

\section{MAXimum LiKelihood Estimator}

In order to keep the discussion more general, all the measured anchor locations and timings are grouped into a vector $\boldsymbol{k}=\left[a_{x 1}, a_{y 1}, \theta_{s 1}, \theta_{01}, \cdots, a_{x L}, a_{y L}, \theta_{s L}, \theta_{0 L}\right]^{T}$, whose covariance matrix is denoted by $C_{k}$ and the true (but unknown) value of $\boldsymbol{k}$ is denoted by $\boldsymbol{k}^{\circ}$.

Dividing both sides of (1) and (2) by $\theta_{s}$, the equations for the $l^{\text {th }}$ anchor are grouped into

$$
\frac{\boldsymbol{T}_{b l}}{\theta_{s}}=\frac{\boldsymbol{T}_{a l}}{\theta_{s l}^{o}}+t_{l} \otimes \mathbf{1}_{2 M \times 1}+\left(\frac{\theta_{0}}{\theta_{s}}-\frac{\theta_{0 l}}{\theta_{s l}}\right) \boldsymbol{E}_{2 M \times 1}+\boldsymbol{n}_{l},
$$

where $\boldsymbol{n}_{l}=\left[n_{l 1}, \bar{n}_{l 1}, \cdots, n_{l M}, \bar{n}_{l M}\right]^{T}$, and

$$
\begin{aligned}
\boldsymbol{T}_{a l} & =\left[-R_{l 1}, \bar{T}_{l 1}, \cdots,-R_{l M}, \bar{T}_{l M}\right]^{T}, \\
\boldsymbol{T}_{b l} & =\left[-T_{l 1}, \bar{R}_{l 1}, \cdots,-T_{l M}, \bar{R}_{l M}\right]^{T}
\end{aligned}
$$

include the time-stamps at the $l^{\text {th }}$ anchor side and Node $B$ side, respectively. Further, stacking the equations for all $L$ anchors, we have $\boldsymbol{T}_{b} / \theta_{s}=\boldsymbol{\mu}+\boldsymbol{n}$, where

$$
\begin{gathered}
\boldsymbol{T}_{a}=\left[\boldsymbol{T}_{a 1}^{T}, \cdots, \boldsymbol{T}_{a L}^{T}\right]^{T}, \boldsymbol{T}_{b}=\left[\boldsymbol{T}_{b 1}^{T}, \cdots, \boldsymbol{T}_{b L}^{T}\right]^{T}, \\
\boldsymbol{\mu}=\boldsymbol{T}_{a} \odot\left(\left[\begin{array}{c}
1 / \theta_{s 1}^{o} \\
\vdots \\
1 / \theta_{s L}^{o}
\end{array}\right] \otimes \mathbf{1}_{2 M \times 1}\right)+\left[\begin{array}{c}
t_{1} \\
\vdots \\
t_{L}
\end{array}\right] \otimes \mathbf{1}_{2 M \times 1} \\
+\frac{\theta_{0}}{\theta_{s}} \boldsymbol{E}_{2 L M \times 1}-\left[\begin{array}{c}
\theta_{01}^{o} / \theta_{s 1}^{o} \\
\vdots \\
\theta_{0 L}^{o} / \theta_{s L}^{o}
\end{array}\right] \otimes \boldsymbol{E}_{2 M \times 1} .
\end{gathered}
$$

Because all the measurement errors are Gaussian and the errors in observed time-stamps are independent of errors in anchor locations and timing parameters, the logarithm of the joint probability density function (PDF) of the observed timestamps, anchor locations and timing parameters is given by

$$
\begin{aligned}
\ln p_{2}\left(\boldsymbol{T}_{a}, \boldsymbol{T}_{b},\left\{\boldsymbol{a}_{l}, \theta_{s l}, \theta_{0 l}\right\}_{l=1}^{L} \mid \boldsymbol{x}, \boldsymbol{\theta},\left\{\boldsymbol{a}_{l}^{o}, \theta_{s l}^{o}, \theta_{0 l}^{o}\right\}_{l=1}^{L}\right) \\
=d-\frac{1}{2}\left(\boldsymbol{T}_{b} / \theta_{s}-\boldsymbol{\mu}\right)^{T} \boldsymbol{C}_{n}^{-1}\left(\boldsymbol{T}_{b} / \theta_{s}-\boldsymbol{\mu}\right) \\
-\frac{1}{2}\left(\boldsymbol{k}-\boldsymbol{k}^{o}\right)^{T} \boldsymbol{C}_{k}^{-1}\left(\boldsymbol{k}-\boldsymbol{k}^{o}\right)
\end{aligned}
$$

where $d$ is a constant and $C_{n}$ is the covariance matrix of the TOA detection error $\boldsymbol{n}$.

As can be seen from (10), the joint PDF includes the terms of anchor location and timing uncertainties. Therefore, 
the ML joint localization and synchronization involves the maximization with respect to location and synchronization parameters of both the unknown node and anchors. Depending on the number of anchors, the problem can be a very high dimensional maximization problem. Even if it is solvable, the ML estimation requires a lot of computational effort. Instead, we propose a low complexity method in the next section.

\section{Proposed Low-COMplexity Least Squares ESTIMATOR}

Dividing both sides of (4) and (5) by $\theta_{s}$, and using the firstorder Taylor series approximation $1 /\left(\theta_{s l}+\Delta \theta_{s l}\right) \approx 1 / \theta_{s l}-$ $\Delta \theta_{s l} / \theta_{s l}^{2}$, we have

$$
\begin{gathered}
-T_{l m} \theta_{1} \approx-\frac{R_{l m}-\theta_{0 l}}{\theta_{s l}}-\theta_{2}+t_{l}+n_{l m} \\
+\frac{R_{l m}-\theta_{0 l}}{\theta_{s l}^{2}} \Delta \theta_{s l}+\frac{1}{\theta_{s l}} \Delta \theta_{0 l}, \\
\bar{R}_{l m} \theta_{1} \approx \frac{\bar{T}_{l m}-\theta_{0 l}}{\theta_{s l}}+\theta_{2}+t_{l}+\bar{n}_{l m} \\
-\frac{\bar{T}_{l m}-\theta_{0 l}}{\theta_{s l}^{2}} \Delta \theta_{s l}-\frac{1}{\theta_{s l}} \Delta \theta_{0 l},
\end{gathered}
$$

where $\theta_{1} \triangleq 1 / \theta_{s}$ and $\theta_{2} \triangleq \theta_{0} / \theta_{s}$ were introduced, and the second order terms of the anchor timing errors were neglected.

Representing $t_{l}$ as $t_{l}=\left\|\boldsymbol{x}-\left(\boldsymbol{a}_{l}+\Delta \boldsymbol{a}_{l}\right)\right\| / c$, moving $t_{l}$ in (11) and (12) to one side and all other terms to the other side, and squaring the equations, (11) and (12) can be formulated into a linear equation as

$$
(\boldsymbol{A}+\Delta \boldsymbol{A}) \boldsymbol{\xi} \approx \boldsymbol{b}+\boldsymbol{v},
$$

where $\boldsymbol{\xi}=\left[\theta_{1}^{2} / 2,\left(\theta_{2}^{2}-\|\boldsymbol{x}\| / c^{2}\right) / 2, \theta_{1} \theta_{2}, \theta_{1}, \theta_{2}, \boldsymbol{x}^{T}\right]^{T}, \boldsymbol{A} \triangleq$ $\left[\boldsymbol{A}_{1}, \boldsymbol{A}_{2}, \cdots, \boldsymbol{A}_{L}\right]^{T}$ and $\boldsymbol{b} \triangleq\left[\boldsymbol{b}_{1}, \boldsymbol{b}_{2}, \cdots, \boldsymbol{b}_{L}\right]^{T}$ with

$$
\begin{gathered}
\boldsymbol{A}_{l}=2\left[\begin{array}{ccccc}
T_{l 1}^{2} & 1 & -T_{l 1} & \frac{-T_{l 1}\left(R_{l 1}-\theta_{0 l}\right)}{\theta_{s l}} & \frac{R_{l 1}-\theta_{0 l}}{\theta_{s l}} \\
\bar{R}_{l 1}^{2} & 1 & -\bar{R}_{l 1} & \frac{-\bar{R}_{l 1}\left(\bar{T}_{l 1}-\theta_{0 l}\right)}{\theta_{s l}} & \frac{\bar{T}_{l 1}-\theta_{0 l}}{\theta_{s l}} \\
& & \vdots & \\
T_{l M}^{2} & 1 & -T_{l M} & \frac{-T_{l M}\left(R_{L M}-\theta_{0 l}\right)}{\theta_{s l}} & \frac{R_{L M}-\theta_{0 l}}{\theta_{s l}} \\
\bar{R}_{l M}^{2} & 1 & -\bar{R}_{l M} & \frac{-\bar{R}_{l M}\left(\bar{T}_{L M}-\theta_{0 l}\right)}{\theta_{s l}} & \frac{\bar{T}_{L M}-\theta_{0 l}}{\theta_{s l}} \\
\boldsymbol{b}_{l}=\left[\begin{array}{c}
\left\|\boldsymbol{a}_{l}\right\| / c^{2}-\left(R_{l 1}-\theta_{0 l}\right)^{2} / \theta_{s l}^{2} \\
\left\|\boldsymbol{a}_{l}\right\| / c^{2}-\left(\bar{T}_{l 1}-\theta_{0 l}\right)^{2} / \theta_{s l}^{2} \\
\vdots \\
\left\|\boldsymbol{a}_{l}\right\| / c^{2}-\left(R_{L M}-\theta_{0 l}\right)^{2} / \theta_{s l}^{2} \\
\left\|\boldsymbol{a}_{l}\right\| / c^{2}-\left(\bar{T}_{L M}-\theta_{0 l}\right)^{2} / \theta_{s l}^{2}
\end{array}\right] .
\end{array}\right.
\end{gathered}
$$

The perturbation terms in (13) are given by

$$
\begin{gathered}
\Delta \boldsymbol{A}=[\mathbf{0}_{2 L M \times 5}, \underbrace{\left.\frac{2}{c^{2}}\left[\Delta \boldsymbol{a}_{1}, \cdots, \Delta \boldsymbol{a}_{L}\right]^{T} \otimes \mathbf{1}_{2 M \times 1}\right]}_{\triangleq \delta \boldsymbol{A}}, \\
\boldsymbol{v} \approx \underbrace{\frac{2}{c^{2}}\left[\boldsymbol{a}_{1}^{T} \Delta \boldsymbol{a}_{1}, \cdots, \boldsymbol{a}_{L}^{T} \Delta \boldsymbol{a}_{L}\right]^{T} \otimes \mathbf{1}_{2 M \times 1}}_{\triangleq \Delta \boldsymbol{b}}+\boldsymbol{e},
\end{gathered}
$$

and the error vector is given by

$$
\begin{aligned}
e=2 \boldsymbol{T}_{e} \odot\left[\boldsymbol{n}+\boldsymbol{T}_{1} \odot\left(\Delta \boldsymbol{\theta}_{s} \otimes \mathbf{1}_{2 M \times 1}\right)\right. & \\
- & \left.\boldsymbol{T}_{2} \odot\left(\Delta \boldsymbol{\theta}_{0} \otimes \mathbf{1}_{2 M \times 1}\right)\right],
\end{aligned}
$$

where

$$
\begin{aligned}
& \boldsymbol{T}_{e}=\boldsymbol{T}_{b} \theta_{1}-\theta_{2} \boldsymbol{E}_{2 L M \times 1}-\boldsymbol{T}_{a} \odot\left(\overline{\boldsymbol{\theta}}_{s} \otimes \mathbf{1}_{2 M \times 1}\right) \\
& +\left(\boldsymbol{\theta}_{0} \odot \overline{\boldsymbol{\theta}}_{s}\right) \otimes \boldsymbol{E}_{2 M \times 1}, \\
& \boldsymbol{T}_{1}=-\boldsymbol{T}_{a} \odot\left[\left(\overline{\boldsymbol{\theta}}_{s} \odot \overline{\boldsymbol{\theta}}_{s}\right) \otimes \mathbf{1}_{2 M \times 1}\right]+\left(\overline{\boldsymbol{\theta}}_{s} \odot \overline{\boldsymbol{\theta}}_{s}\right) \otimes \boldsymbol{E}_{2 M \times 1}, \\
& \boldsymbol{T}_{2}=\overline{\boldsymbol{\theta}}_{s} \otimes \boldsymbol{E}_{2 M \times 1},
\end{aligned}
$$

with $\Delta \boldsymbol{\theta}_{s}=\left[\Delta \theta_{s 1}, \cdots, \Delta \theta_{s L}\right]^{T}, \Delta \boldsymbol{\theta}_{0}=\left[\Delta \theta_{01}, \cdots, \Delta \theta_{0 L}\right]^{T}$, $\boldsymbol{\theta}_{0}=\left[\theta_{01}, \cdots, \theta_{0 L}\right]^{T}$ and $\overline{\boldsymbol{\theta}}_{s} \triangleq\left[1 / \theta_{s 1}, \cdots, 1 / \theta_{s L}\right]^{T}$.

Since the perturbation $\Delta \boldsymbol{A}$ is unobservable, the observation model of (13) is

$$
A \xi=b+v .
$$

Equation (17) can be interpreted as a linear system with model error in the model coefficient matrix $\boldsymbol{A}$. The generalized total least square (GTLS) technique can be employed to provide consistent estimates of $\boldsymbol{\xi}$ [14]. To derive the GTLS solution for problem (17), the coefficient matrix $\boldsymbol{H} \triangleq \boldsymbol{A}$ is partitioned into $\boldsymbol{H}=\left[\begin{array}{ll}\boldsymbol{H}_{1} & \boldsymbol{H}_{2}\end{array}\right]$, where $\boldsymbol{H}_{1}$ is the first five columns of $\boldsymbol{H}$, which are free of error, and $\boldsymbol{H}_{2}$ is the last two columns of $\boldsymbol{H}$, which are subject to errors. The GTLS problem [13] seeks to find a minimum norm approximation of the weighted error by

$$
\begin{aligned}
& \min _{\left[\begin{array}{ll}
\hat{\boldsymbol{H}}_{2} & \boldsymbol{\epsilon}
\end{array}\right]}\left\|\left(\left[\boldsymbol{H}_{2} \boldsymbol{\epsilon}\right]-\left[\hat{\boldsymbol{H}}_{2} \hat{\boldsymbol{\epsilon}}\right]\right) \boldsymbol{C}_{U}^{-1 / 2}\right\|_{F} \\
& \text { subject to } \quad \widehat{\boldsymbol{\epsilon}} \in \mathcal{R}\left(\left[\begin{array}{ll}
\boldsymbol{H}_{1} & \hat{\boldsymbol{H}}_{2}
\end{array}\right]\right),
\end{aligned}
$$

where $\boldsymbol{\epsilon} \triangleq \boldsymbol{b}+\boldsymbol{v}, \boldsymbol{U} \triangleq[\delta \boldsymbol{A}, \boldsymbol{v}]$ and $\boldsymbol{C}_{U}$ is the covariance matrix of $\boldsymbol{U}^{T}$ and is shown to be

$$
\begin{aligned}
\boldsymbol{C}_{U} & =\frac{8 M \sigma_{a}^{2}}{c^{4}}\left[\begin{array}{ccc}
L & 0 & \sum_{l=1}^{L} a_{x l} \\
0 & L & \sum_{l=1}^{L} a_{y l} \\
\sum_{l=1}^{L} a_{x l} & \sum_{l=1}^{L} a_{y l} & \frac{c^{4} T r\left(\boldsymbol{C}_{v}\right)}{8 M \sigma_{a}^{2}}
\end{array}\right] \\
& \triangleq\left[\begin{array}{cc}
\boldsymbol{C}_{A} & \boldsymbol{c}_{A v} \\
\boldsymbol{c}_{A v}^{T} & c_{v}
\end{array}\right],
\end{aligned}
$$

where $C_{A}$ is a $2 \times 2$ matrix, $c_{A v}$ is a $2 \times 1$ vector, $c_{v}$ is a scalar and $\boldsymbol{C}_{v}$ is the covariance matrix of $\boldsymbol{v}$ given by

$$
\begin{aligned}
\boldsymbol{C}_{v} & =\frac{4 \sigma_{a}^{2}}{c^{4}}\left(\boldsymbol{a}_{x} \boldsymbol{a}_{x}^{T}+\boldsymbol{a}_{y} \boldsymbol{a}_{y}^{T}\right) \otimes \mathbf{1}_{2 M \times 2 M} \\
& +4\left(\boldsymbol{T}_{e} \boldsymbol{T}_{e}^{T}\right) \odot\left[\sigma_{n}^{2} \boldsymbol{I}_{2 L M}+\sigma_{s}^{2} \boldsymbol{T}_{1} \boldsymbol{T}_{1}^{T}+\sigma_{o}^{2} \boldsymbol{T}_{2} \boldsymbol{T}_{2}^{T}\right],
\end{aligned}
$$

with $\boldsymbol{a}_{x}=\left[a_{x 1}, \cdots, a_{x L}\right]^{T}$ and $\boldsymbol{a}_{y}=\left[a_{y 1}, \cdots, a_{y L}\right]^{T}$. Due to space limitation, details of the derivations of $C_{U}$ and $C_{v}$ are not presented here.

From [14], the closed-form solution of the GTLS problem (18) is

$$
\hat{\boldsymbol{\xi}}_{G T L S}=\left(\boldsymbol{H}^{T} \boldsymbol{H}-\gamma^{2} \tilde{\boldsymbol{C}}_{A}\right)^{-1}\left(\boldsymbol{H}^{T} \boldsymbol{\epsilon}-\gamma^{2} \tilde{\boldsymbol{c}}_{A v}\right),
$$

where $\tilde{\boldsymbol{C}}_{A}=\operatorname{diag}\left[\mathbf{0}_{5 \times 5}, \boldsymbol{C}_{A}\right], \tilde{\boldsymbol{c}}_{A v}=\left[\begin{array}{ll}\mathbf{0}_{1 \times 5}, & \boldsymbol{c}_{A v}^{T}\end{array}\right]^{T}$ and $\gamma$ is the smallest generalized singular value of matrix pair $\left(\left[\begin{array}{ll}\boldsymbol{\epsilon}\end{array}\right], \operatorname{diag}\left[\mathbf{0}_{5 \times 5}, \boldsymbol{C}_{U}^{1 / 2}\right]\right)$. 
After the GTLS solution (21) is obtained, the relationship between elements of $\boldsymbol{\xi}$ can also be exploited. To carry out the refinement, the estimation covariance matrix of $\hat{\boldsymbol{\xi}}_{G T L S}$ is needed. Defining $\boldsymbol{\Gamma} \triangleq\left(\boldsymbol{H}^{T} \boldsymbol{H}-\gamma^{2} \tilde{\boldsymbol{C}}_{A}\right)^{-1}$, we have

$$
\begin{aligned}
\hat{\boldsymbol{\xi}}_{G T L S}-\boldsymbol{\xi} & =\boldsymbol{\Gamma}\left(\boldsymbol{H}^{T} \boldsymbol{\epsilon}-\gamma^{2} \tilde{\boldsymbol{c}}_{A v}\right)-\boldsymbol{\Gamma} \boldsymbol{\Gamma}^{-1} \boldsymbol{\xi} \\
& =\boldsymbol{\Gamma}\left[\left(\boldsymbol{H}^{T} \boldsymbol{\epsilon}-\gamma^{2} \tilde{\boldsymbol{c}}_{A v}\right)-\left(\boldsymbol{H}^{T} \boldsymbol{H}-\gamma^{2} \tilde{\boldsymbol{C}}_{A}\right) \boldsymbol{\xi}\right] \\
& \approx \boldsymbol{\Gamma} \boldsymbol{H}^{T}\left(\boldsymbol{\epsilon}-\boldsymbol{H}^{T} \boldsymbol{\xi}\right) \\
& =\boldsymbol{\Gamma} \boldsymbol{H}^{T} \boldsymbol{v} .
\end{aligned}
$$

The approximation in (22) is due to the fact that $\gamma^{2} \tilde{\boldsymbol{c}}_{A v}$ and $\gamma^{2} \tilde{\boldsymbol{C}}_{A}$ are very small and can be approximated by zero. With (22), the covariance matrix of $\hat{\boldsymbol{\xi}}_{G T L S}$ is

$$
\begin{aligned}
\boldsymbol{C}_{G T L S} & =E\left\{\left(\hat{\boldsymbol{\xi}}_{G T L S}-\boldsymbol{\xi}\right)\left(\hat{\boldsymbol{\xi}}_{G T L S}-\boldsymbol{\xi}\right)^{T}\right\} \\
& \approx \boldsymbol{\Gamma} \boldsymbol{H}^{T} \boldsymbol{C}_{v} \boldsymbol{H} \boldsymbol{\Gamma}^{T} .
\end{aligned}
$$
by

The relationship between elements of $\boldsymbol{\xi}$ can be represented

$$
G \omega=\xi,
$$

where $\boldsymbol{\omega}=\left[\theta_{1}, \theta_{2}, \boldsymbol{x}^{T}\right]^{T}$, and

$$
\boldsymbol{G}=\left[\begin{array}{c}
\tilde{\boldsymbol{G}} \\
\boldsymbol{I}_{4}
\end{array}\right], \tilde{\boldsymbol{G}}=\frac{1}{2}\left[\begin{array}{cccc}
\theta_{1} & 0 & 0 & 0 \\
0 & \theta_{2} & -\frac{x}{c^{2}} & -\frac{y}{c^{2}} \\
\theta_{2} & \theta_{1} & 0 & 0
\end{array}\right] .
$$

With the covariance matrix in (23) and exploiting the constraints between $\boldsymbol{\xi}$ in (24), we have

$$
\hat{\boldsymbol{\omega}}_{C G T L S}=\left(\hat{\boldsymbol{G}}^{T} \boldsymbol{C}_{G T L S}^{-1} \hat{\boldsymbol{G}}\right)^{-1} \hat{\boldsymbol{G}}^{T} \boldsymbol{C}_{G T L S}^{-1} \hat{\boldsymbol{\xi}}_{G T L S},
$$

where $\hat{G}$ is obtained by putting the estimated values of $x, y$, $\theta_{1}$ and $\theta_{2}$ from $\hat{\boldsymbol{\xi}}_{G T L S}$ in (21) to the corresponding variables in $\boldsymbol{G}$.

Remark 1: The final estimates of $\theta_{s}$ and $\theta_{0}$ are obtained from the estimates $\hat{\theta}_{1}$ and $\hat{\theta}_{2}$ in (26) by $\hat{\theta}_{s}=1 / \hat{\theta}_{1}$ and $\hat{\theta}_{0}=\hat{\theta}_{2} / \hat{\theta}_{1}$.

Remark 2: In implementation, the closed-form solution in (21) is typically not used to solve the GTLS problem because it is generally numerically unstable. A numerically stable and efficient procedure for obtaining the GTLS solution, which does not involves any matrix inversion, is presented in [14].

\section{Cramer-Rao Lower Bound}

In order to provide a performance reference for the proposed estimator, the CRLB is derived in this section.

The parameters of interest are grouped into $\Phi=$ $\left[x, y, \theta_{s}, \theta_{0}\right]^{T}$. With the joint PDF in (10), the CRLB of $\mathbf{\Upsilon}=\left[\boldsymbol{\Phi}^{T}, \boldsymbol{k}^{o T}\right]^{T}$, is derived as [12]

$$
\operatorname{CRLB}(\boldsymbol{\Upsilon})=-\left[E\left\{\frac{\partial^{2} \ln p_{2}}{\partial \boldsymbol{\Upsilon} \partial \boldsymbol{\Upsilon}^{T}}\right\}\right]^{-1}=\left[\begin{array}{ll}
\boldsymbol{S}_{1} & \boldsymbol{S}_{2} \\
\boldsymbol{S}_{2}^{T} & \boldsymbol{S}_{3}
\end{array}\right]^{-1}
$$

where

$$
\begin{aligned}
& \boldsymbol{S}_{1}=-E\left\{\frac{\partial^{2} \ln p_{2}}{\partial \boldsymbol{\Phi} \partial \boldsymbol{\Phi}^{T}}\right\}=\left\{\frac{\partial \boldsymbol{\zeta}}{\partial \boldsymbol{\Phi}}\right\}^{T} \boldsymbol{C}_{n}^{-1}\left\{\frac{\partial \boldsymbol{\zeta}}{\partial \boldsymbol{\Phi}}\right\} \\
& \boldsymbol{S}_{2}=-E\left\{\frac{\partial^{2} \ln p_{2}}{\partial \boldsymbol{\Phi} \partial \boldsymbol{k}^{o T}}\right\}=\left\{\frac{\partial \boldsymbol{\zeta}}{\partial \boldsymbol{\Phi}}\right\}^{T} \boldsymbol{C}_{n}^{-1}\left\{\frac{\partial \boldsymbol{\mu}}{\partial \boldsymbol{k}^{o}}\right\} \\
& \boldsymbol{S}_{3}=-E\left\{\frac{\partial^{2} \ln p_{2}}{\partial \boldsymbol{k}^{o} \partial \boldsymbol{k}^{o T}}\right\}=\left\{\frac{\partial \boldsymbol{\mu}}{\partial \boldsymbol{k}^{o}}\right\}^{T} \boldsymbol{C}_{n}^{-1}\left\{\frac{\partial \boldsymbol{\mu}}{\partial \boldsymbol{k}^{o}}\right\}+\boldsymbol{C}_{k}^{-1}
\end{aligned}
$$

with $\boldsymbol{\zeta}=\boldsymbol{T}_{b} / \theta_{s}-\boldsymbol{\mu}$. The partial derivatives in the above equation are derived as

$$
\frac{\partial \boldsymbol{\zeta}}{\partial \boldsymbol{\Phi}}=\left[\boldsymbol{B}_{1}, \boldsymbol{B}_{2}\right], \quad \frac{\partial \boldsymbol{\mu}}{\partial \boldsymbol{k}^{o}}=\operatorname{diag}\left[\boldsymbol{D}_{1}, \cdots, \boldsymbol{D}_{L}\right],
$$

where

$$
\begin{aligned}
& \boldsymbol{B}_{1}= \frac{1}{c^{2}}\left[\left(\boldsymbol{x}-\boldsymbol{a}_{1}^{o}\right) / t_{1}, \cdots,\left(\boldsymbol{x}-\boldsymbol{a}_{L}^{o}\right) / t_{L}\right]^{T} \otimes \mathbf{1}_{2 M \times 1}, \\
& \boldsymbol{B}_{2}= {\left[\frac{1}{\theta_{s}^{2}}\left(-\boldsymbol{T}_{b}+\theta_{0} \boldsymbol{E}_{2 L M \times 1}\right), \frac{1}{\theta_{s}} \boldsymbol{E}_{2 L M \times 1}\right], } \\
& \boldsymbol{D}_{l}= {\left[\left(\boldsymbol{a}_{l}^{o}-\boldsymbol{x}\right)^{T} \otimes \mathbf{1}_{2 M \times 1} /\left(c^{2} t_{l}\right),\right.} \\
&\left.\quad\left(-\boldsymbol{T}_{a l}+\theta_{0 l} \boldsymbol{E}_{2 M \times 1}\right) / \theta_{s l}^{o 2},-\boldsymbol{E}_{2 M \times 1} / \theta_{s l}^{o}\right] .
\end{aligned}
$$
by

Using the matrix inversion lemma, the CRLB for $\boldsymbol{\Phi}$ is given

$$
\operatorname{CRLB}(\boldsymbol{\Phi})=\boldsymbol{S}_{1}^{-1}+\boldsymbol{S}_{1}^{-1} \boldsymbol{S}_{2}\left(\boldsymbol{S}_{3}-\boldsymbol{S}_{2}^{T} \boldsymbol{S}_{11}^{-1} \boldsymbol{S}_{2}\right)^{-1} \boldsymbol{S}_{2}^{T} \boldsymbol{S}_{1}^{-1} .
$$

From the above expression, we can see that the first term in (30) is the CRLB for $\boldsymbol{\Phi}$ when the anchor positions and timings are accurate, and the second term represents the increase in CRLB of $\boldsymbol{\Phi}$ when there are uncertainties in the anchor positions and synchronization parameters.

\section{Simulation Results and Discussions}

In this section, simulation results are presented to verify the effectiveness of the proposed scheme. In the simulations, there are four anchors located at $(-50,40) \mathrm{m},(-40,-90) \mathrm{m}$, $(90,-60) \mathrm{m}$ and $(20,70) \mathrm{m}$. The sensor node to be located and synchronized is located at $(10,30) \mathrm{m}$. The number of timestamp exchange round is set to $M=4$. The clock skew and clock offset of Node $B$ are 1.00005 and 5 ns, respectively. The unit of all time-stamps is nanosecond. The variances of uncertainties in anchor location, time skew an time offset are set to $1 / \sigma_{a}^{2}=40 \mathrm{~dB}, 1 / \sigma_{s}^{2}=80 \mathrm{~dB}$ and $1 / \sigma_{o}^{2}=20 \mathrm{~dB}$. The mean square error (MSE) of the location is defined as $E\left\{(x-\hat{x})^{2}+(y-\hat{y})^{2}\right\}$. All simulations are average of 1000 independent runs.

The performance of the proposed GTLS estimator is compared to the ML estimator (ignoring the anchor uncertainties) presented in [6], the CRLB in Section V, and the CRLB with accurate anchor locations and timings. From Figures 2 and 3, it can be seen that the MSE of the proposed GTLS estimator can touch the CRLB for both localization and time synchronization. For the ML estimator ignoring anchor uncertainties, it is obvious that it fails to provide accurate estimates 
of location and time offset, with performance far from the CRLB. Furthermore, there are observable degradations in the CRLB when the the anchors are inaccurate compared to that for accurate anchors.

\section{CONCLUSIONS}

In this paper, we investigated the similarities between localization and time synchronization in wireless sensor networks and proposed a unified framework to jointly solve the localization and time synchronization problems where there are uncertainties in both anchor locations and timings.

The problem was formulated into a linear equation with errors in the model matrix. A generalized total least squares based scheme was proposed to estimate the node location and timing. Cramer-Rao lower bound (CRLB) was also derived for the proposed joint estimator. Simulation results showed that the mean square error performance of the proposed estimator can attain the CRLB.

\section{REFERENCES}

[1] R. Poovendran, C. Wang, and S. Roy (eds), Secure localization and time synchronization for wireless sensor and ad hoc networks, Springer, 2006.

[2] A. Swami, Q. Zhao, Y. Hong, and L. Tong (eds), Wireless sensor networks: Signal processing and communications perspective, Wiley, 2007.

[3] G. Mao, B. Fidan, and B. Anderson, "Wireless sensor network localization techniques," Computer Networks, vol.51, no.10, pp.2529-2553, Jul. 2007.

[4] B. Sundararaman, U. Buy, and A. Kshemkalyani, "Clock synchronization for wireless sensor networks: a survey," Ad Hoc Networks, vol,3, no.3, pp.281-323, May 2005.

[5] H. Oliveira, E. Nakamura, A. Loureiro, "Localization in time and space for sensor networks," in Proc. 21st International Conf. on Advanced Info. Net. and App., pp.539-546, May 2007.

[6] J. Zheng and Y. C. Wu "Localization and Time Synchronization in Wireless Sensor Networks: a Unified Approach," in Proc. IEEE Asia Pacific Conf. on Circuits and Sys. (APCCAS), Nov.30 - Dec.3, 2008, Macao, China.

[7] S. Ganeriwal, R. Kumar and M. B. Srivastava, "Timing-syn protocol for sensor networks," SenSys 03, Nov 2003.

[8] K. Noh, Q. M. Chaudhari, E. Serpedin and B. W. Suter, "Novel clock phase offset and skew estimation using two-way timing message exchanges for wireless sensor networks," IEEE Trans. on Communications, vol.55, no.4, pp.766-777, 2007.

[9] N. Freris, P. Kumar, "Fundamental limits on synchronization of affine clocks in networks," 46th IEEE Conf. on Decision and Ctrl, Dec. 2007.

[10] B. Denis, J. Pierrot and C. Abou-Rjeily, "Joint distributed synchronization and positioning in UWB ad hoc networks using ToA," IEEE Trans. on Microwave Theory and Tech., vol. 54, no. 4, Apr. 2006.

[11] IEEE Standard 802.15.4a, Aug. 2007.

[12] S. Kay, Fundamentals of Statistical Signal Processing: Estimation Theory, Englewood Cliffs, NJ: Prentice-Hall, 1993.

[13] S. Van Huffel and J. Vandewalle, The total least-squares problem: computational aspects and analysis, Volumne 9 of Frontier in Applied Mathematics, Philadelphia, PA: SIAM, 1991.

[14] S. Van Huffel and J. Vandewalle, "Analysis and properties of the generalized total least squares problem $A X \approx B$ when some or all columns in $A$ are subject to error," SIAM J. Matrix Anal. Appl. vol.10, no. 3, pp.294-315, July 1989.

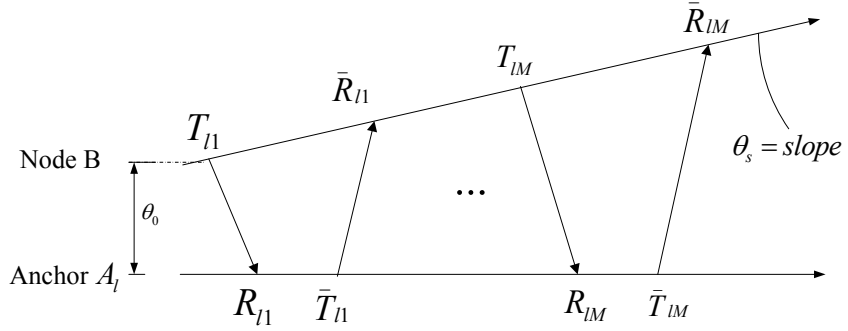

Fig. 1. Two-way time-stamp exchange between Node $B$ and the $l^{\text {th }}$ anchor $A_{l}$.

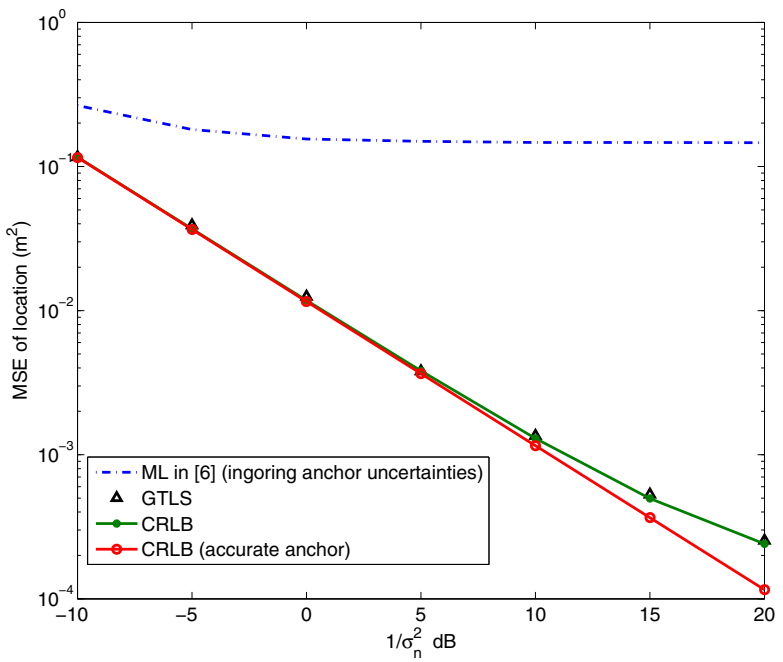

Fig. 2. MSE of location versus TOA detection error variance $1 / \sigma_{n}^{2}$, with anchor location error variance $1 / \sigma_{a}^{2}=40 \mathrm{~dB}$, anchor timing error variances $1 / \sigma_{s}^{2}=80 \mathrm{~dB}$ and $1 / \sigma_{o}^{2}=20 \mathrm{~dB}$.

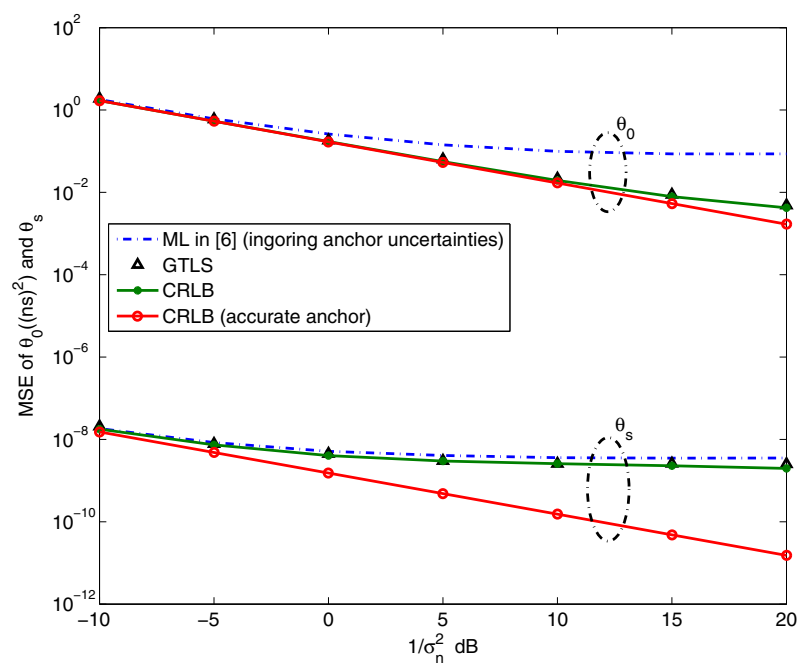

Fig. 3. MSE of $\theta_{s}$ and $\theta_{0}$ versus TOA detection error variance $1 / \sigma_{n}^{2}$, with anchor location error variance $1 / \sigma_{a}^{2}=40 \mathrm{~dB}$, anchor timing error variances $1 / \sigma_{s}^{2}=80 \mathrm{~dB}$ and $1 / \sigma_{o}^{2}=20 \mathrm{~dB}$. 